\title{
Local Observables and Renormalization in Dirac-Segal Approach to QFT
}

\author{
Andrey Losev
}

Received: 29 May 2014 / Accepted: 9 September 2014 / Published online: 31 October 2014

(C) School of Mathematical Sciences, University of Science and Technology of China and Springer-Verlag Berlin Heidelberg 2014

\begin{abstract}
We explain the Dirac-Segal approach to quantum field theory. We study local observables in this approach and the theory of deformations. We found out that this theory of deformation in the second-order coincides with the renormalization of the same theory, would it be considered in Polyakov approach. We conjecture that it is still true to all orders.
\end{abstract}

Keywords Quantum field theory $\cdot$ Segal axioms $\cdot$ Renormalization

Mathematics Subject Classification $81 \mathrm{~T} 16$

\section{Introduction}

Let us recall an issue of the cut-off that appears is standard approaches to the quantum field theory.

The functional integral approach [1] to quantum field theory that appeared in 1950s was considered as a main approach starting from 1970s. However, in most cases the integral itself has no mathematical meaning, and only its formal perturbative expansion is sensible. Moreover, even this expansion in almost all cases suffers from ultraviolet divergences, and in practical use we have to do renormalization procedure [2]. To do this we choose linear structure on the space of fields (that naturally exists for connections and sections of vector bundles but is absolutely unnatural for scalar fields that are maps from the space-time to some manifold) and on the space-time. In such case we may speak about the momentum of the fields, and we restrict desirable integral over the

\footnotetext{
A. Losev ( $\square)$

Scientific Research Institute of System Development and National Research University

"Higher School of Economics", Moscow, Russia

e-mail: aslosev2@gmail.com
} 
infinite-dimensional space of fields to the integral over finite-dimensional subspace of fields with momenta smaller than so-called cut-off momenta. So the integral could be computed; however, it diverges when the cut-off tends to infinity. Therefore, we consider some parameters in the action functional to be dependent on the cut-off and consider only such dependencies that the integral has the finite limit when cut-off goes to infinity.

Another approach to quantum field theory based on axioms for correlator was proposed by Wightman [3]. This approach is nonperturbative in nature; however, the deformation theory for solutions to these axioms was not developed.

The third approach was proposed by Polyakov [4]. In this approach the functional integral is considered as a result of phase transition of test partition function on a lattice. This approach as a Wightman approach allows nonperturbative treatment; however, it contains the distance between points of the lattice (we assume that lattice is embedded into Euclidean space) as an analog of the cut-off parameter. Moreover, the variables on the lattice are often quite different from the fields that are used in description of emerging theory, and the fact of phase transition is hard to prove. Nevertheless, this approach led to axioms of conformal field theory in terms of correlators, thus, generalizing the Wightman axioms. Moreover, in some cases, nontrivial correlators were defined and computed. However, its deformation theory has problems. It is clear that the tangent space is given by local observables of dimension 2. At the same time consideration of the second order of deformation led to divergencies when moving points started to approach to each other. In order to make sense of the second order, one had to define a cut-off, saying that the minimal distance between points is bounded from below by a positive scale $a$ that was thought of as a distance between points on the lattice. The deformed theory contained divergencies as $a \rightarrow 0$, so it needs renormalization (that may be achieved by subtracting the divergent part of the correlator), but it is unclear how to proceed further for the third order in perturbation theory.

In this article we will see that the notion equivalent to renormalization naturally appears in the Dirac-Segal approach to QFT. Namely, it has a geometrical meaning of construction of the tangent space to deformed theory.

\section{Dirac-Segal Approach to QFT}

In this section we define the nonperturbative approach to QFT that I called Dirac-Segal approach. After the definition I comment the origin of the name of this approach.

Let $X$ be a $d$-dimensional manifold with boundary, and $\Gamma=\cup_{i=1}^{N} \Gamma_{i}$ its boundary, consisting of $N(d-1)$-dimensional components. Manifold $X$ is assumed to be equipped with some kind of geometrical data that we denote by $g$-it could be metric, complex structure, almost complex structure, connection, or everything else that locally is described by a finite set of functions.

The Dirac-Segal approach associates with each boundary component $\Gamma_{i}$ a Hilbert space $V_{i}$ (that can be heuristically considered as a space of boundary conditions) and to the manifold $X$-the element $I$ (that replaces "functional integral")

$$
I(X, g) \in V_{1} \otimes \ldots V_{N} \otimes F u n\left(\operatorname{Geom}_{X}\right)
$$


where $F u n_{X}\left(\operatorname{Geom}_{X}\right)$ is the space of functions on the space of geometrical data on $X$.

The first axiom is that if $X$ is a disjoint union of two manifolds $X_{1}$ and $X_{2}$, then

$$
I(X, g)=I\left(X_{1}, g_{1}\right) \otimes I\left(X_{2}, g_{2}\right)
$$

The main axiom is the cutting axiom that deals with the cutting of $X$ along the $(d-1)$-dimensional submanifold $\Gamma_{0}$ into a manifold $X^{c}$, such that the geometry $g$ induces geometry $g^{c}$. It reads

$$
I(x, g)=<I\left(X^{c}, g^{c}\right)>,
$$

where

$$
<>V_{0} \otimes V_{0} \otimes V_{1} \otimes \ldots V_{N} \rightarrow V_{1} \otimes \ldots V_{N}
$$

is induced by the scalar product on $V_{0}$, the space of boundary conditions associated with $\Gamma_{0}$ ("integration over common boundary conditions")

As an example, consider 1-dimensional theories with metric as a geometric data. The 1-dimensional manifolds with two boundaries are intervals; the main object is an operator $I(t) \in \operatorname{End}(V) \otimes C^{\infty}(R)$, where $t$ is the length of the interval, and the main axiom states

$$
I\left(t_{1}+t_{2}\right)=I\left(t_{1}\right) \cdot I\left(t_{2}\right) \text {, i.e., } I(t)=\exp (t H) .
$$

If we consider $H$ as an Hamiltonian, we get Dirac form of the evolution operator that explains the Dirac name in the approach. It is remarkable that there is no Plank constant in the expression for $I$ since it is internal quantum expression while Plank constant expresses the distance from the quantum theory to its classical limit.

Segal [5] introduced these set of axioms in description of the 2-dimensional theories, and in his example, the geometrical data were complex structure. It should be noted that Atiyah [6] used the same set of axiom for theories with no geometrical data at all, i.e., topological theories, but we would like to concentrate on physical theories in this article.

\section{Local Observables in Dirac-Segal Formulation of QFT}

There is an important difference between Dirac-Segal formulation of QFT and two other formulations. In Wightman formulation the notion of local observable is just built in the system of axioms. In functional integral formulation this notion comes just from local functions of fundamental fields (however, such observables have to be renormalized by subtracting from the local expressions, other local expressions depending on the cut-off). Similar things happen in Polyakov's approach.

In the Dirac-Segal approach, origin of local observables is very different. They are not the part of the fundamental data. However, it is possible to define them as a derived object as follows. 
Consider a point $P$ on the $d$-dimensional space $X$ and form a set of disks $D_{r}$ centered at $P$ and of the radius $r$ for all sufficiently small positive values of $r$. Here we assume that the geometric data on the space $X$ contain metric. Consider the set of spaces with the disk cut out, i.e., $X_{r}=X-D_{r}$. Such spaces would have one additional boundary for $d>1$ and two boundaries for $d=1$. It is clear that $I\left(X_{r}, g\right)$ takes values in $V_{r} \otimes V_{1} \otimes \ldots V_{N}$ for $d>1$ and in $V_{r} \otimes V_{r} \otimes V_{1} \otimes \ldots V_{N}$ for $d=1$. Consider families $u_{r} \in V_{r}$ (for $\left.d>1\right)$ and $u_{r} \in V_{r} \otimes V_{r}$ ( for $d=1$ ), such that there is a limit which we call $I\left(X, O_{P}\right)$ :

$$
\lim _{r \rightarrow 0}<u_{r}, I\left(X_{r}\right)>=I\left(X, O_{P}\right)
$$

Here pairing means pairing in the space $V_{r}$ for $d>1$ and pairing in the space $V_{r} \otimes V_{r}$ for $d=1$. We will call such families good families. Note that there are good families that have zero limit - we will call them null families. The space of local observables is a coset of the space of good families over the space of null families. From main axiom one can show that this definition is independent of the space $X$, as a space $X$ one may take just a disk of radius $R$ around $P$.

Let us consider as an example 1-dimensional theory with a finite-dimensional space of states $V$. Then any local observable may be represented by a constant family, i.e., an operator $O \in V \otimes V$. Moreover, in this case

$$
I\left(\text { interval, } T, O_{t}\right)=\exp ((T-t) H) O \exp (t H)
$$

that is a well-known formula in quantum mechanics.

Note that in the case of 2-dimensional conformal theories, the definition has to be modified since there is no metric to play with. In this case we should proceed as follows. We pick up a local holomorphic coordinate at a point $P$ that we will call $Z_{P}$ and consider family of disks $D_{r}$ defined by $\left|Z_{P}\right|<r$. In such definition it becomes clear that the very notion of local observable depends on the choice of local coordinate - a well-known phenomena in 2-dimensional CFT.

It is important to note that given a QFT, the computation of its space of local observable is a nontrivial problem. However, there is a trick that helps analyze this problem. Suppose that we have already found several local observables, say $O^{a}$ where $a$ belongs to some set $A$. Then we may consider the following family:

$$
u_{r}^{a b}=I\left(D_{r}, O_{0}^{a}, O_{Q}^{b}\right) \in V_{r},
$$

such that distance between $Q$ and zero equals to $r / 2$. This family may not be a good family, typically that the limit in question would blow up as $1 / r^{\Delta}$ for positive $\Delta$. In this case we may consider an improved family that will turn out good, namely $r^{\Delta} u_{r}^{a b}$. Such family defines a local observable that may already be in the span of observables from our set, in this case the constructed observable would have the form $C_{c}^{a b} O^{c}$. In the opposite case we just enlarge the set $A$ of local observables. After we had completed this step, we may analyze the following family:

$$
u_{r}^{a b}-C_{c}^{a b} I\left(D_{r}, O_{0}^{c}\right),
$$


this family may diverge as $r^{-\tilde{\Delta}}$, with $\tilde{\Delta}<\Delta$. We repeat this procedure, and at the end of the day we find the well-known expression that is historically called OPE:

$$
I\left(D_{r}, O_{0}^{a}, O_{Q}^{b}\right)=C_{c}^{a b} r^{-\Delta} I\left(D_{r}, O_{0}^{c}\right)+\tilde{C}_{c}^{a b} r^{-\tilde{\Delta}} I\left(D_{r}, O_{0}^{c}\right)+\cdots
$$

\section{Local Observables as a Tangent Space to the Space of Deformations of Dirac-Segal QFT}

Local observables are important because they are describing the tangent space to the space of deformations of QFT that preserve vector spaces associated to boundaries. If we believe in the functional integral approach, we may think of local observables as terms in Lagrangian of the theory. This not quite true, the space of local observables is bigger than the space of possible terms in Lagrangian. For example, consider the Gaussian model. There are vortex local observables, and theory may be deformed by them. In order to see this we have to go to dual formulation. At the same time we may deform the theory by adding to Lagrangian periodic functions of the fundamental field. It could also be described by deformation of the Lagrangian written in original variables. However, if we deform the theory by both vortex observables and periodic functions, we would fail to find the Lagrangian description of such theory. This argument is one of the several reasons that make Lagrangian description of QFT, a bit outdated.

In order to get an idea for the description of the space of deformation of QFT, let us consider the general 1-dimensional QFT (quantum mechanics) with finite-dimensional space of states. As we have shown above, such theories are parametrized by an operator $H$ (Hamiltonian). One can easily find out that in the first order in deformation parameter, the deformed Dirac-Segal map looks as follows:

$$
\exp (T(H+\delta H))-\exp (T H)=\int_{0}^{T} \mathrm{~d} t \exp ((T-t) H) \delta H \exp (t H)=\int \mathrm{d} t I\left(T, \delta H_{t}\right)
$$

that has a meaning of the integral of the local observable $\delta H$ inserted at point $t$ over the full space, i.e., the interval $[0, T]$.

One can easily show that 1-dimensional case may be generalized to the multidimensional case, namely, the tangent space of deformations of Dirac-Segal QFT is given by local observables, and all first-order deformations have the form

$$
\delta I(X)=\int_{P \in X} I\left(X, O_{P}\right) \mu_{P}
$$

where $\mu_{P}$ is a measure on the space $X$ that depends on the nature of the local observable. In particular, we may have to enlarge the set of geometrical data needed to describe a theory.

We have already observed this phenomena in 1-dimensional case. Let us start with topological quantum mechanics, i.e., with the system with zero Hamiltonian (that has no geometrical data) and deform it to nonzero Hamiltonian. In this case we extend 
empty space of geometrical data by the space of 1 forms, in particularly, by a 1-form $\mathrm{d} t$ (that may be considered as a measure on the interval constructed with the help of the metric on the interval). The same phenomena would happen in deformation of conformal theory (that depends only on the conformal class of metric, i.e., complex structure) into a massive theory (where space of geometrical data consists of metrics).

The expression in (9) really solves linearized axioms (1) and (2) of QFT. In order to show the inverse, i.e., that all deformation has form of (9), we should represent the manifold $X$ as a "net" filled by many disks. Due to main axiom deformation of the theory is a sum of deformations associated to disks that brings up the integral in the right-hand side of (9). The smallness of disks leads to insertion of local observable at the place of a disk. It is quite instructive to apply as an exercise this argument to a 1-dimensional theory, i.e., to an interval.

\section{Deformations Beyond the First Order and Renormalization}

From the presentation we gave it is clear how to go to the second order in deformation of QFT. We just need to consider the second deformation as a first-order deformation to the deformed theory. Here we find an interesting phenomena: local observables of the original theory fail to be the local observables to the deformed one (if dimension of the space-time is greater than one). Are we surprised? Not at all, really, if we consider axioms (1) and (2) as equations on variables $I$, we see that the axiom (1) is nonlinear, and we do not expect to have a linear space of solutions (that is the space of all theories). Therefore, the space of local observables forms a tangent sheaf. But is it a bundle, or do all fibers have the same dimensions?

It is instructive to consider similar case not in the infinite-dimensional space of variables $I$ but in finite-dimensional geometry. Consider $R^{k}$ and nonlinear equation there, say

$$
x_{1}^{2}+\cdots+x_{k}^{2}=\vec{x}^{2}=1,
$$

we know that the space of solutions is a $k-1$ dimensional sphere $S^{k-1}$, but let us pretend that we do not know it in advance. Let as assume that we know a solution $\vec{x}_{*}=(1,0, \ldots, 0)$. Then the tangent vectors are solution to linear equations:

$$
\delta \vec{x} \cdot \vec{x}_{*}=0
$$

and they form $(k-1)$-dimensional space.

Let us pick some tangent vector $\vec{v}$ that is solution to Eq. (11), i.e., that is orthogonal to $\vec{x}_{*}$. The deformed solution is $\vec{x}+\epsilon \vec{v}$, and deformed equation for tangent vector is

$$
\vec{x}_{*} \cdot \delta \vec{x}+\epsilon \vec{v} \cdot \delta \vec{x}=0
$$

We see that old tangent vectors in general are not tangent vectors anymore due to the second term in (12). However, we can always correct it by adding term proportional to $\vec{x}_{*}$ and find

$$
\delta \vec{x}=\vec{w}-\epsilon(\vec{v} \cdot \vec{w}) \vec{x}_{*}+\epsilon \vec{z}
$$

for any two vectors $\vec{w}, \vec{z}$ that are orthogonal to vector $\vec{x}_{*}$. 
Thus, we see that the space of tangent vectors in the deformed theory is the same while there is an arbitrariness in the choice of solution to Eq. (12). Moreover, taking $\vec{z}=0$ we define a connection on the tangent space; however, this connection has curvature (for $k>2$ ), and it is impossible to get a natural coordinates of the space of solutions to main equation compatible with the connection.

We presented here this simple example from differential geometry, because very similar things happen with deformations of QFT.

Really, if the theory is deformed with the local observable $O^{v}$ then in the deformed theory with the main object $I^{\epsilon v}$ the contraction with the good (at $\epsilon=0$ ) family (that corresponded to local observable $O^{w}$ ) gives

$$
u_{r}^{w} I^{\epsilon v}\left(X_{r}\right)=\epsilon C_{y}^{w v} r^{d-\Delta} I\left(X, O_{P}^{y}\right),
$$

where $r^{0}$ should be understood as $\log r$.

The divergent terms have to be corrected like in (13) and the analog of (13) looks as follows:

$$
u_{r}^{w, \epsilon}=u_{r}^{w}-\epsilon C_{y}^{w v} r^{d-\Delta} u_{r}^{y}+\epsilon u_{r}^{z} .
$$

First of all, we note that there is no obstruction for deformation of local observable; this means that they form a bundle. However, it may happen that deformed observable naturally couples to more general geometrical data than that original one. Such phenomena should be called anomaly, and conformal anomaly in 2-dimensional CFT deformed by the marginal observable is of this type. We see that the space of possible geometrical data has to be separately studied, and we will return to this question in another paper.

What is more important is that second-order renormalization that we have in DiracSegal approach coincides at the level of algebraic expressions with those that we could get using coordinate cut-off in Polyakov approach.

We conclude with the conjecture that properly defined renormalization in Polyakov approach is just the perturbation theory in Dirac-Segal approach, where the cut-off scale plays the role of parameter $r$, appearing in the definition of a local observable.

\section{References}

1. Feynman, R., Hibbs, A.: Quantum Mechanics and Path Integrals. McGraw-Hill, New York (1965)

2. Collins, J.: Renormalization. Cambridge University Press, Cambridge (1984)

3. Streater, RF., Wightman, AS.: PCT, spin and statistics, and all that. Princeton, Benjamin, New York (1964)

4. Polyakov, A.: Gauge Fields and Strings. Harwood Academic Publishers, Chur (1986)

5. Segal, G.: The definition of conformal field theory. Differential Geometric Methods in Theoretical Physics. Nato Adv. Sci. Inst. Ser. C Math. Phys. Sci, vol. 250, pp. 165-171. Kluwer Academic Publishers, Dordrecht (1988)

6. Atiyah, M.: New invariants of three and four dimensional manifolds. In: Proceedings of Symposia in Pure Mathematics, 48, AMS, 48,285 